\title{
Interference RNA in immune-mediated oral diseases - minireview
}

\author{
RAGHU DHANAPAL ${ }^{l}$, SREEPALLAVI SOMASUNDARAPANDIAN ${ }^{l}$, SOSROSENO WIHASKORO ${ }^{l}$, \\ RANGANATHAN KANNAN ${ }^{2}$ GOVIND RAJKUMAR ${ }^{3}$, RAMASAMY CHIDAMBARAM ${ }^{1}$
}

${ }^{1}$ Aimst Dental Institute, Aimst University, Bedong, Malaysia

${ }^{2}$ Ragas Dental College, Chennai, Malaysia

${ }^{3}$ Vishnu Dental College, Bhimavaram, Malaysia

\begin{abstract}
Immune-mediated oral disorders are characterised by their chronicity, and some are refractory to treatment. Interference RNA ( $i R N A$ ) has been implicated in the underlying mechanism of such immune-mediate oral and refractory inflammatory oral diseases. iRNA-based understanding of the mechanism in these diseases may help to produce non-invasive diagnostic methodologies and treatment modalities of such drug non-responsive diseases. Oral lesions in these immune-mediated diseases can precede the occurrence of lesions in other regions of the body. The early diagnosis and treatment of these drug non-responsive diseases might benefit the patient by reducing chronicity and probably even resolving the disease. This aim of the present minireview is to give an overview of the possible implications of iRNA on the pathogenesis, diagnosis, and treatments of immune-mediated and inflammatory oral diseases. The manuscript can form the framework for research on iRNA in these immune-mediated oral disorders.
\end{abstract}

Key words: RNA interference, Immune disorders, oral manifestations, RNAi therapies.

(Centr Eur J Immunol 2017; 42 (3): 301-304)

\section{Introduction}

Andrew Fire and Craig Mello retain the credit for discovering interference RNAs (iRNA). A simple experiment in Caenorhabditis elegans on muscle twitching protein expression, with sense and antisense sequence of the transcripts, led to the discovery of iRNA [1]. The interference RNAs control gene expression by post-transcriptional activity and cleaving homologue transcripts [1, 2]. Furthermore, iRNA can be used to regulate the expression of proteins that are not easily accessed by traditional pharmacological approaches, such as molecules lacking ligand-binding domains or proteins that share a high degree of structural homology. Thus, iRNA-based therapeutic approaches are especially appealing to achieve a high degree of specificity and to target molecules that are considered to be "drug non-responsive diseases" [3, 4]. Common chronic oral disease are refractory to medical treatment; this group mostly encompasses of inflammatory and immune mediated diseases. The role of interference RNA in such drug non-responsive chronic diseases with oral manifestations is briefly discussed herein, which is the first of its kind.

Theoretically, literature acknowledges iRNA as small noncoding (nc) RNAs. It comprises mall interference RNA (siRNA), Piwi interacting RNA (piRNA), and micro RNA
(miRNA). Short hairpin RNA (shRNA) are synthetically created interference RNAs [5, 6].

siRNAs are short linear RNAs. They consist of nucleotide sequences of 21-25 bp length derived from cleavage of a double-stranded RNA. They block translation by forming a complex with Ago (Argonaute proteins help in interference) and by endonuclease activity causing cleavage of transcripts (process of slicing) [7, 8]. Systemic therapeutic administration of siRNA's into human tumours has been a significant advance in tumour treatment [9].

piRNAs form complexes of RNA protein by their interactive action with piwi proteins [10]. piwi proteins act by targeting transposons [11]. piRNAs commonly function in germ cells, mainly during spermatogenesis [10]. They silence genes by epigenetic and post-transcriptional mechanisms [12].

miRNAs are a highly conserved, small, single-stranded, non-coding RNAs. miRNAs bind to the partial complimentary site of mRNA in three different regions 3' or 5' and the coding region, and cause post-translational repression $[6,13]$. The miRNA has diverse cellular functions, which include control of cellular functions, embryogenesis, and immune function [14-18]. miRNA expression and function can also be influenced by inflammation and an immunological challenge [19].

Correspondence: Raghu Dhanapal, Aimst Dental Institute, Aimst University, B3/B12 Staff Quarters, AIMST Dental Institute,

Aimst University, Semeling, 08100 Bedong, Malaysia, e-mail: raghudhanapal@ gmail.com

Submitted: 12.07.2016; Accepted: 16.08.2016 
In contrast to the above-mentioned interference RNA, shRNAs are artificially created iRNAs that require viral vectors or plasmids to be transcribed into the cell or can also deliver siRNA [20]. The effectiveness of shRNAs is that they are heritable, stable, and more potent in mammalian cells, on par with siRNA [21].

The iRNA subtypes may play an important role in the treatment outcome of immune-mediated oral diseases. Further communications are tailored considering the role of different iRNAs in immune-mediated oral diseases and common inflammatory oral diseases. Oral lesions are a common manifestation of certain immunologically mediated diseases and autoimmune disorders, and these diseases may be untreatable with medications [22, 23]. In autoimmune disorders such as pemphigus the oral lesions are the first manifestation of the disease process in 50-90\% of patients [24]. Lesions of oral lichen planus have a greater propensity for recurrence and resistance to treatment compared to the cutaneous lesions [25]. Thus, the dilemma the oral physician faces in such scenarios could be resolved by target therapy.

There is previous literature on the role of interference RNA in oral diseases, but this article, to the best of our knowledge, is the first to focus on the possible role of immune-mediate oral diseases.

\section{Allergic contact stomatitis}

Allergic contact stomatitis tends to manifest as lichen planus-like lesions in response to certain materials such as amalgam, gold, and acrylic. The lesions do not resolve despite removal of the offending agent in $5 \%$ of cases. Under such circumstances, interference RNA can play a vital role [22]. In addition, the TNF- $\alpha$ expression is also increased in animal models in contact allergies. The TNF- $\alpha$ level is suppressed by siRNA-mediated targeting of TNF- $\alpha$ receptors $[26,27]$. This therapeutic application can form the basis of treatment in 5\% cases of persistent allergic stomatitis lesions.

\section{Oral lichen planus}

The analysis of this mucocutaneous disease exhibits an altered mRNA profile associated with T-cell activation [28]. Erosive oral lichen planus, which has a potential malignant lesion, has been controversial. The promoter of miRNA exhibits methylation in OLP patients, and the expression profile of miRNAs from oral lichen planus (OLP) patient tissue when compared to tissue from healthy individuals revealed that 11 pairs of miRNA and mRNA associated with OLP were functionally associated with the potentially malignant nature of OLP $[29,30]$. In lichen planus, the miRNAs upregulated were mi-21, mi-31, mi-132, and mi-155. These miRNAs are also commonly over-expressed in precancer and cancer, thus emphasising the malignant potential of lichen planus [30]. Oral lesions are more persistent compared to skin lesions in lichen planus [25]. iRNA-based target therapy of such refractory oral lesions could benefit the patient.

\section{Aphthous and Bechet's disease}

Alteration in T-cell regulation (Treg cells) has been implicated in the pathogenesis of both aphthous and Bechet's disease. The cardinal factors behind the altered regulatory mechanism include change functioning of the CD4+CD25+ Treg cells, abnormal variants of Toll-like receptors, abnormal cytokine cascade, and production of immunomodulatory enzyme [Indoleamine2,3-dioxygenase (IDO)]. Decrease in IDO leads to failure of mucosal immune tolerance in aphthous ulcer patients [31]. shRNA -based suppression of IDO has been effective in cancer therapy and could possibly be implicated in the treatment of these immune-mediated diseases. miRNA profile in Treg cells has antiproliferative or proapoptotic activity for suppression of T-cells, which suggests its valuable therapeutic potential in oral aphthous ulcers in the prevention of recurrent lesions, a common concern of both patients and clinicians [32].

\section{Sjögren's syndrome}

Sjögren's syndrome is frequently identified by the dentist on the basis signs and symptoms of xerostomia [33]. Ago, GW182, and Rck/p54 are proteins that aid in the functioning of miRNAs and are localised in the cytoplasm as GW bodies (GWB). In patients with Sjögren's syndrome serum analysis revealed auto-antibodies that were generated against GWB. $31 \%$ of the patients had these auto-antibodies, denoting altered miRNA functioning [34]. miR-768-3p and miR-574 from saliva have been used as biomarkers for analysis of inflammation and salivary gland dysfunction in Sjögren's syndrome patients [35], thus implicating the role of iRNA as an effective non-invasive diagnostic tool.

\section{Pemphigus}

Oral lesions are the initial signs in the majority of pemphigus cases, later progressing to cutaneous lesions [24]. Inhibition of EGFR by shRNA (short hairpin) in human keratinocytes leads to a blockage of the pemphigus auto-antibody-induced acantholysis. This is due to prevention of the desmoglein internalisation and keratin intermediate filament retraction. PV IgG (pemphigus vulgaris antibody)-induced vesicle or blister formation was prevented in a mouse model by inhibition of EGFR both by using EGFR inhibitors and shRNA [36]. Therefore, iRNA-based therapeutic intervention could have a good prognostic outcome in pemphigus lesions that are difficult to treat. 


\section{Inflammatory oral lesions}

Periodontitis and periapical lesions are the most common inflammatory lesions affecting the oral cavity. The interference RNA can have a profound effect on the suppression of these lesions.

\section{Periodontal diseases and periapical lesions}

Periodontal diseases and periapical lesions are the most common causes of tooth extraction and are associated with loss of supporting alveolar bone. Certain cases of periodontal diseases are refractory to any treatment modalities, and iRNA may be an effective tool. Mitogen-activated protein kinase (MAPK) in chronic periodontitis has been implicated in the activation of inflammatory cytokines [37]. MAPK is a molecule that has been associated with difficulty in developing an inhibitor. Hence, in chronic periodontitis, inhibition of bone loss by siRNA to MAPK in palatal regions of artificially-induced bone loss was due to decreased osteoclastogenesis and inflammatory infiltrate [38]. iRNA has a profound effect on new bone formation in areas of bone loss. iRNA in osteoblastic cells increased bone formation via activating BMP-2 signalling on alkaline phosphatase, SMAD phosphorylation, and increased expression of osteocalcin and Runx-2 [39]. Furthermore, siRNA-targeted therapy on TNF- $\alpha$ has prevented osteolysis in animal models of periapical lesions. shRNA-mediated knockdown of gene expression of Atp6i gene (which forms a subunit of osteoclast proton pump) prevented periapical inflammation and bone resorption in an animal model [40]. Previous reports suggest an important role of iRNA in the pathogenesis of oral inflammatory lesions and their therapeutic implications.

\section{Conclusions}

Within the limitations of the undertaken review, it is clear that iRNA may provide an insight into the pathogenesis of these immune-mediated oral diseases and refractory inflammatory oral diseases. iRNA-based analyses may prove to be an ideal tool not only for diagnosis but also for the treatment of some of these diseases. Furthermore, these molecules can also be a predictive marker in the prognosis and lead to a favourable outcome for these diseases. Further studies are required to delineate the exact role of iRNA in immune-mediated oral diseases.

The authors declare no conflict of interest.

\section{References}

1. Fire A, Xu S, Montgomery MK, et al. (1998): Potent and specific genetic interference by double-stranded RNA in Caenorhabditis elegans. Nature 391: 806-811.
2. Hui L, Muller F, George AC (2013): MicroRNAs and other non-coding RNAs as targets for anticancer drug development. Nat Rev Drug Discov 12: 847-865.

3. Tiemann K, Rossi JJ (2009): RNAi-based therapeutics-current status, challenges and prospects. EMBO Mol Med 1: 142-151.

4. Ma J, Dong C, Ji C (2010): MicroRNA and drug resistance. Cancer Gene Therapy 17: 523-531.

5. Ranganathan K, Sivasankar V (2014): MicroRNAs-biology and clinical applications. J Oral Maxillofac Pathol 18: 229-234.

6. Carthew RW, Sontheimer EJ (2009): Origins and Mechanisms of miRNAs and siRNAs. Cell 136: 642-655.

7. Femke S, Alessia B, Isabelle CK, et al. (2010): Hairpin RNA induces secondary small interfering RNA synthesis and silencing in trans in fission yeast. EMBO Rep 11: 112-118.

8. Willkomm S, Restle T (2015): Conformational Dynamics of Ago-Mediated Silencing Processes. Int J Mol Sci 16: 1476914785.

9. Pecot CV, Calin GA, Coleman RL (2011): RNA interference in the clinic: challenges and future directions. Nat Rev Cancer 11: 59-67.

10. Kandhavelu M, Lammi C, Buccioni M, et al. (2009): Existence of snoRNA, microRNA, piRNA characteristics in a novel non-coding RNA: $x$-ncRNA and its biological implication in Homo sapiens. J Bioinformatics Seq Anal 1: 31-40.

11. Sienski G, Donertas D, Brennecke J (2012): Transcriptional silencing of transposons by Piwi and maelstrom and its impact on chromatin state and gene expression. Cell 151: 964-980.

12. Hsueh-Yen K and Haifan L (2014): PIWI proteins and their interactors in piRNA biogenesis, germline development and gene expression. Natl Sci Rev 1: 205-218.

13. Felekkis K, Touvana E, Stefanou C, et al. (2010): MicroRNAs: a newly described class of encoded molecules that play a role in health and disease. Hippokratia 14: 236-240.

14. Yi R, O'Carroll D, Pasolli HA, et al. (2006): Morphogenesis in skin is governed by discrete sets of differentially expressed microRNAs. Nat Genet 38: 356-362.

15. Cheng AM, Byrom MW, Shelton J, et al. (2005): Antisense inhibition of human miRNAs and indications for an involvement of miRNA in cell growth and apoptosis. Nucleic Acids Res 33: 1290-1297.

16. Yekta S, Tabin CJ. Bartel DP (2008): MicroRNAs in the Hox network: An apparent link to posterior prevalence. Nat Rev Genet 9: 789-796.

17. Guo SQ, Lu J, Schlanger R, Zhang H, Wang JY (2010): MicroRNA miR-125a controls hematopoietic stem cell number. PNAS 107: 14229-14234.

18. Hu YL, Fong S, Largman C, et al. (2010): HOXA9 regulates miR-155 in hematopoietic cells. Nucleic Acids Res 16: 54725478.

19. Ryan MO'C, Dinesh SR, Aadel AC, et al. (2013): Physiological and pathological roles for microRNAs in the immune system. Immunol Rev 253: 112-128.

20. Siyun X, Yongsheng X, Li L, et al. (2014): Three new shRNA expression vectors targeting the CYP3A4 coding sequence to inhibit its expression. Acta Pharm Sin B 4: 350-357.

21. Patrick JP, Amy AC, Emily B, et al. (2002): Short hairpin RNAs (shRNAs) induce sequence-specific silencing in mammalian cells. Genes Dev 16: 948-958.

22. Cowan GM, Lockey RF (2014): Oral manifestations of allergic, infectious, and immune-mediated disease. J Allergy Clin Immunol Pract 2: 686-696.

23. Bascones-Martínez A, García-García V, Meurman JH, et al. (2015): Immune-mediated diseases: what can be found in the oral cavity? Int J Dermatol 54: 258-270. 
24. Shamim T, Varghese VI, Shameena PM, et al. (2008): Pemphigus vulgaris in oral cavity: Clinical analysis of 71 cases. Med Oral Pathol Oral Cir Bucal 13: E622-E626.

25. Nico MM, Fernandes JD, LourenÇo SV (2011): Oral lichen planus. An Bras Dermatol 86: 633-641.

26. Li W, Zhao Y, Xu X, et al. (2015): Rebamipide suppresses TNF-a mediated inflammation in vitro and attenuates the severity of dermatitis in mice. FEBS J 2015; 282: 2317-2326.

27. Verma N, Chaudhury I, Kumar D, et al. (2009): Silencing of TNF-alpha receptors coordinately suppresses TNF-alpha expression through NF-kappaB activation blockade in THP-1 macrophage. FEBS Lett 583: 2968-2974.

28. Li X, Li J, Yang Y, et al. (2013): Differential gene expression in peripheral blood $\mathrm{T}$ cells from patients with psoriasis, lichen planus, and atopic dermatitis. J Am Acad Dermatol 69: e235-e243.

29. Dang J, Bian YQ, Sun JY, et al. (2013): MicroRNA-137 promoter methylation in oral lichen planus and oral squamous cell carcinoma. J Oral Pathol Med 42: 315-321.

30. Volker G, Jochen H, Yahya A, et al. (2013): Disease-Associated miRNA-mRNA Networks in Oral Lichen Planus. PLoS One 8: e63015.

31. Lewkowicz N, Lewkowicz P, Dzitko K, et al. (2008): Dysfunction of $\mathrm{CD} 4+\mathrm{CD} 25$ high $\mathrm{T}$ regulatory cells in patients with recurrent aphthous stomatitis. J Oral Pathol Med 37: 454-461.

32. Huang TT, Yen MC, Lin CC, et al. (2011): Skin delivery of short hairpin RNA of indoleamine 2,3 dioxygenase induces antitumor immunity against orthotopic and metastatic liver cancer. Cancer Sci 102: 2214-2220.

33. Al-Hashimi (2001): The management of Sjögren's syndrome in dental practice J Am Dent Assoc 132: 1409-1417.

34. Bhanji RA, Eystathioy T, Chan EK, et al. (2007): Clinical and serological features of patients with autoantibodies to GW/P bodies. Clin Immunol 125: 247-256.

35. Alevizos I, Alexander S, Turner RJ, et al. (2011): MicroRNA expression profiles as biomarkers of minor salivary gland inflammation and dysfunction in Sjogren's syndrome. Arthritis Rheum 63: 535-544.

36. Bektas M, Jolly PS, Berkowitz P, et al. (2013): A pathophysiologic role for epidermal growth factor receptor in pemphigus acantholysis. J. Biol. Chem 288: 9447-9456.

37. Qiyan L, Michael SV, Keith LK (2012): MAPK Usage in Periodontal Disease Progression. J Signal Transduct 2012: 308943.

38. Li Q, Yu H, Zinna R, Martin K, et al. (2011): Silencing MAP kinase-activated protein kinase-2 arrests inflammatory bone loss. J Pharmacol Exp Ther 336: 633-642.

39. Dabra S, Chhina K, Soni N, et al. (2012): Tissue engineering in periodontal regeneration: A brief review. Dent Res J 96: 71-80.

40. Ma J, Chen W, Zhang L, et al. (2013): RNA interference-mediated silencing of Atp6i prevents both periapical bone erosion and inflammation in the mouse model of endodontic disease. Infect Immun 81: 1021-1030. 